\title{
A multi-objective evolutionary scheme for control points deployment in intelligent transportation systems
}

\author{
Martin Luther Mfenjou ${ }^{1}$, Ado Adamou Abba Ari ${ }^{2}$, Arouna Ndam Njoya ${ }^{3}$, Kolyang ${ }^{4}$, Wahabou Abdou ${ }^{5}$, \\ Abdelhak Mourad Gueroui ${ }^{6}$ \\ ${ }^{1,2,4}$ LaRI Lab, University of Maroua, Maroua, Cameroon, Afrika \\ ${ }^{2,6}$ LI-PaRAD Lab, Université Paris Saclay, University of Versailles Saint-Quentin-en-Yvelines, Versailles cedex, France \\ ${ }^{1,3}$ Department of Apply Mathematics and Computer Science, LASE Lab, University of Ngaoundéré, Ngaoundere, \\ Cameroon \\ ${ }^{5}$ LE2I Lab - UMR CNRS 6306, University of Burgundy, Dijon cedex, France
}

\begin{tabular}{|c|c|}
\hline Article Info & ABSTRACT \\
\hline Article history: & \multirow{11}{*}{$\begin{array}{l}\text { One of the problems that hinder emergency in developing countries is the problem } \\
\text { of monitoring a number of activities on inter-urban roadway networks. In the litera- } \\
\text { ture, the use of control points is proposed in the context of these countries in order } \\
\text { to ensure efficient monitoring, by ensuring a good coverage while minimizing the in- } \\
\text { stallation costs as well as the number of accidents across these road networks. In this } \\
\text { work, we propose an optimal deployment of these control points from several opti- } \\
\text { mization methods based on some evolutionary multi-objective algorithms: the Non } \\
\text { dominated sorting genetic algorithm-II (NSGA-II); the multi-objective particle swarm } \\
\text { optimization (MOPSO); the strength pareto evolutionary algorithm-II (SPEA-II); and } \\
\text { the pareto envelope based selection algorithm-II (PESA-II). We performed the tests } \\
\text { and compared these deployments using pareto front and performance indicators like } \\
\text { the spread and hypervolume and the inverted generational distance (IGD). The results } \\
\text { obtained show that the NSGA-II method is the most adequate in the deployment of } \\
\text { these control points. }\end{array}$} \\
\hline Received Jan 14, 2020 & \\
\hline Revised Oct 6, 2020 & \\
\hline Accepted Oct 17, 2020 & \\
\hline & \\
\hline & \\
\hline Multi-objective evolutionary & \\
\hline Algorithms & \\
\hline Roadway network & \\
\hline Deployment & \\
\hline Performance evaluation & \\
\hline
\end{tabular}

This is an open access article under the blueCC BY-SA license.

Control points

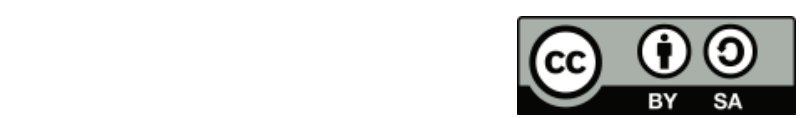

\section{Corresponding Author:}

Ado Adamou Abba Ari

LaRI Lab, University of Maroua, P.O. Box 814 Maroua, Cameroon

Email: adoadamou.abbaari@gmail.com

\section{INTRODUCTION}

Today, monitoring activities through road transport networks remain a serious problem for both developed and developing countries since road accidents cause huge losses. Shoukrallah and Rifaat [1] noted that around the world, we have about 1.2 million people killed on the road in 2008 at an estimated total cost of $\$ 518$ billion, and 50 million people are injured in road accident. Moreover Jadaan Khair [2] in their work on a comparative analysis of road safety in developed and developing countries showed that $90 \%$ of deadly accidents are caused in developing countries while these countries only have 54\% of the global vehicle rate. The emergence of intelligent transportation systems (ITS) together with the new generation of mobile networks contribute to a serious reduction of the death rate on the roads [3-6]. These systems exploit technological advances of telecommunications to facilitate communication among users, precisely monitor activities in road network [7]. The implementation of such concepts and systems become necessary for developing countries.

In the case of the developing countries, more work are aimed at laying the groundwork for road 
networks oversight models for inter-urban transport. For instance, based on some factors such as smart parking, city-wide traffic monitoring, as well as public transportation information services, Gohar et al. [8] proposed a big data analytics architecture for ITS. Moreover, Silva et al. [9] studied smart cities in the context of ITS while reviewing their features and characteristics as well as some challenges and opportunities. Furthermore, sustainable smart city schemes and framework have been developed in order to enhance ITS users mobility in some cities of developing countries $[10,11]$. However, these models should consider the infrastructural realities of these countries.

Thus, Mfenjou et al. [4] proposed a methodology that aims at contextualizing an ITS by taking into account the infrastructural realities. In [12], the authors proposed a monitoring communication architecture for developing countries' inter-urban road networks based on control points. They then introduced a deployment model for these control points according to the following criteria: minimizing the installation cost of these points, maximizing network coverage and considering areas where accidents are frequently occurring in developing countries' inter-urban road network. This deployment has been performed by the Non-Dominated sorting genetic algorithm II (NSGA-II). For the experimented scenarios, authors end up at the pareto front that helps decision-makers to take appropriate decisions.

Several works focus on the issues of monitoring and regulation transport systems of developing countries. For the reduction of traffic violation such as speed violation, Tarapiah et al. [13] used GPS/GPRS to propose a smart on-board transportation management system that can be attached to a vehicle. This system can help to track a vehicle depending on their position using Google map. This system offers innovations in terms of vehicle tracking in developing countries. No information is sent between the decision-making and the road users in relation to the dissemination of information on road traffic.

Moreover, the proposed works in [14] exploited smartphone sensors to classify the paved and unpaved road as well as road anomaly detection. This solution can only be used to detect obstacles across the road transport network. It may have an impact incidents that occur on the roads, but without communicating obstacles to other users. On the another hand, Patel et al. [15], propose a survey on ITS using the internet of things (IoT). It presents the advantages and inconvenient of proposed approaches in the use of the IoT for ITS. Therefore, in the context of developing countries, technologies used on the IoT could be exploited for the regulation or monitoring of activities through inter-urban road networks. Likewise, the work of Mfenjou et al. [12] focuses on monitoring activities through an inter-urban road network by setting up an architecture of communication based on control points in order to enhance the regulation of inter-urban road networks in developing countries. The proposed deployment was done using an optimization method: NSGA-II. It would be interesting to use several optimization methods to see which one could give better solutions. The following section provides a literature review of some optimization methods.

The purpose of this work is to perform the deployment of the control points using some optimization methods including the Non dominated sorting genetic algorithm-II (NSGA-II), the multi-objective particle swarm optimization (MOPSO), the strength pareto evolutionary algorithm -II (SPEA-II), and the pareto envelope based selection algorithm-II (PESA-II). Indeed, the use of several optimization method's leads to an evaluation of it thanks to the performance indicators. For comparison purpose, among the performance indicators available in the literature, we adopted the hypervolume, inverted generational distance (IGD) and spread.

The rest of the paper is organized as follows: In Section 2, we review some optimization methods based on evolutionary algorithms and their applications in the context of nodes or control points deployment. As for section 3, we propose a methodology to be adopted to carry out the deployments of the controls points and to analyze the obtained results. Concerning the section 4 , we present the results of the pareto front of the non dominated solutions resulting from the used methods of optimization. Then, we evaluate these optimization methods thanks to some performance indicators. Finally, section 5 summarizes, concludes and introduce the future work.

\section{OVERVIEW OF SOME OPTIMIZATION METHODS}

\subsection{Multi-objective genetics algorithms}

Introduced by John Holland [16], genetics algorithms are among other the most used evolutionary algorithms. Whether in the context of single objective problems or multi objective problems, they are based on:

- Encoding [17]: It is at this step that we define how we want to represent the information that we must 
process. The coding can then be binary, octal, hexadecimal, real, permutation and tree encoding.

- Selection [18, 19]: At this level of genetic algorithm, it finds the best individuals for the mating process in such a way that the offsprings produced are better than in the previous population. Depending on how the best individual is sought, several methods of selection exist, but the most known are: the roulette wheel selection, the linear rank selection, the exponential rank selection, the tournament, the truncation selection.

- Crossover [20, 21]: Here, the operation consists in merging two parents in order to generate a third new individual. There are also many variants of crossover: single point crossover, uniform crossover, $\mathrm{N}$ point crossover, three parent crossover, and arithmetic crossover.

- Mutation [22, 23]: Here, the algorithm must generate solutions that are overall likely solutions. We also have several types of mutations: inversion mutation, insert mutation, scramble mutation, flip mutation, swap mutation, interchanging mutation, reversing mutation, uniform mutation, creep mutation.

- Evaluation [16]: To check whether the individual (if he optimizes function), the objective function is used. Otherwise, we go back to the selection.

- Replacement [22-25]: To become the best individual, it would reflect the related problem posed initially in the reality. There are many types of replacement: generational replacement, steady state replacement, elitism, delete n-last, delete n, weak parent replacement, both parents replacement, random replacement.

We are interested in problem solving by multi-objective algorithms. They are grouped into three classes [26]; aggregating function, population-based approaches, and pareto-based approaches. In this work, we are specially interested on nondominated sorting genetic algorithm II (NSGA-II), strength pareto evolutionary algorithm-II (SPEA-II), and pareto envelope based selection algorithm-II (PESA-II).

\subsection{Nondominated sorting genetic algorithm II (NSGA-II)}

This is one of the most used optimization methods. Its efficiency is recognized since compared to some optimization methods its complexity is $\mathrm{O}\left(\mathrm{m} N^{2}\right)$. It is based on a classification of individuals in several levels [27]; it uses the elitist approach that gives it the ability to save the best solutions found from previous generations; it uses a calculation function of the crowding distance. This calculation makes it possible to choose the best solutions in a pareto front. This ensures a uniform dispersion of solutions on the pareto front.

\subsection{Strength pareto evolutionary algorithm II (SPEA-II)}

The SPEA-II [28] is an evolutionary multi objective algorithm that has an improved fitness assignment scheme that takes into account for each individual how many individuals it dominates and how many individuals it is dominated by. Moreover, the SEPA-II has a nearest neighbor density estimation scheme that allows a more precise guidance of the search process. Also, this evolutionary algorithm has an archive truncation scheme that guarantees the preservation of boundary solutions.

\subsection{Pareto envolope based selection algorithm II (PESA-II)}

It is also another optimization method based on evolutionary multi-objective algorithms. It uses a better technique for selecting individuals. Here, instead of associating an individual with a selective fitness, a selective fitness will be assigned to the hyperboxes in which the space is currently occupied by individuals in the approximation of the pareto frontier [29]. This method is more sensitive to ensure a better spread along the pareto frontier than methods based on individual selection.

\subsection{Particle swarm optimization (PSO)}

Particle swarm optimization (PSO) is developed by Kennedy and Eberthart [30]. The swarm of particles are possible solutions, optimization consists of browsing the space of search to find the global optimum. Eberhart and Shi [31] proposed the global version of the PSO. In their proposal, every particle of the swarm keeps tracks of its coordinates in the problem space that are associated with the best solution it achieved: this solution is known as pbest. The best solution value of all the swarm particle is called gbest. The aims of PSO is therefore to improve the gbest value. In fact, it consists at each time step, in updating the velocity. Each particle toward its pbest and gbest locations. This algorithm is based on the social behavior of animals that evolve swarm like schools of fish and flights of birds groups. It is a dynamic that springs from the local information, rules and memory of each individual that forms the basis of their movement while maintaining 
cohesion with the rest of the animals in the group. From this information and rules, we quote: "stay close to the other individual", "go in the same direction", "go at the same speed as the others". The movement of a particle is therefore influenced by the component of inertia, the cognitive component and the social component. Concerning the component of inertia, the particle tends to follow the current direction. About the cognitive component, a particle tends to move towards the best site by which he already passed. Finally, as for the social component, a particle tends to rely on the experience of its neighbors. The swarm is then composed of a set of particles each of which has a position (solution vector) and a speed. Any particle also has a memory allowing him to remember his best performance and that of his neighbors. The swarm of particles are possible solutions, optimization consists of browsing the search space to find the global optimum.

PSO has been improved either by the modification of certain parameters, have the confinement of particles [32], the constriction factor [33], the inertia weight [34], the neighborhood topology [30]. Among the improved versions, we have fully informed particle swarm (FIPS) [35], 5TRIBES algorithm [36], PSO and hybridization [37-39] and Cooperative PSO [40]. Hence, this section allowed us to review the optimization methods that we used in the rest of this work. The following section proposes the approach we consider for the evaluation of the deployment of control points.

\section{OUR PROPOSED SCHEME}

\subsection{Problem formulation and design goal}

The architecture presented in Figure 1 adapts to the technological and infrastructural context of these countries. It is based on control points. Two types have been defined: the treatment control points (TrCP) and the relay control points (RCP). The treatment control point ( $\mathrm{TrCP}$ ) have to main goal of processing the information collected by Relay Control Point. The information concern the behavior of vehicles traveling and disturbances in the inter-urban transport network.

As done in [12], the implementation of control points is based on two objectives: to minimize installation costs and to maximize the coverage in the inter-urban road network. The multi objective formulation given as shown in (5) associated with this type of control point according the objectives $F_{1}$ and $Z_{1}$ as shown in (1) and (2) as well as constraints $C_{1}$ and $C_{2}$ given in (3) and (4).

$-F_{1}$ : the objective function that represents the deployment cost of TrCPS.

$$
F_{1}=\sum_{i \in N \subseteq \mathbb{N}} \sum_{j \in S \subseteq E} c_{i, j} x_{i, j}
$$

- $Z_{1}$ allows the maximization of the coverage in the inter-urban roadway network. Indeed, $Z_{1}$ guarantees that all vehicles passing through the road network is visible and registered by at least one $\operatorname{TrCP}$.

$$
Z_{1}=\sum_{i \in N \subseteq \mathbb{N}} \sum_{j \in S \subseteq E} \sum_{\substack{k \\ k, l \notin S}} m_{k, j} m_{j, l} x_{i, j}
$$

where $m_{i, j}$ is a boolean variable that is equal to 1 if there is an edge starting from $i$ to $j$ and 0 otherwise.

The corresponding constraints $C_{1}$ and $C_{2}$ are given as shown in (3) and (4).

$$
\begin{gathered}
C_{1}: \sum_{i \in N \subseteq \mathbb{N}} x_{i, j} \leq n_{1}^{*}, \forall j \in S \subseteq \\
C_{2}: \sum_{i \in N \subseteq \mathbb{N}} \sum_{j \in S \subseteq E} x_{i, j} \leq n_{2}^{*}, x_{i, j} \in\{0,1\}, \forall i \in N, \forall j \in S \subseteq E \\
\left\{\begin{array}{l}
\text { Minimize } F_{1} \\
\text { Maximize } Z_{1} \\
\text { subjected to } C_{1} \text { and } C_{2}
\end{array}\right.
\end{gathered}
$$


The used notations in the modeling of the optimization problem of TrCPs are given in Table 1.

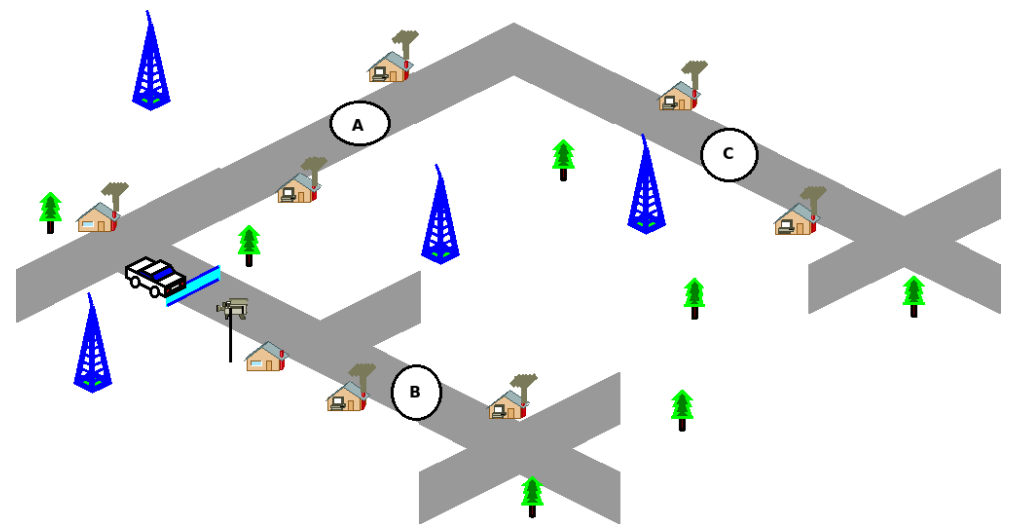

Figure 1. Architecture of communication a control points

Table 1. Notations

\begin{tabular}{lc}
\hline Variable & Notation \\
\hline Deployment cost to build on a given location $j \operatorname{TrCP} P_{i}$ & $c_{i, j}>0$ \\
A decision variable for opening or not the $\operatorname{TrCP} P_{i}$ at location $j$ & $x_{i, j} \in\{0,1\}$ \\
Number of TrCPs candidates & $N$ \\
Set of admissible TrCP positions & $S \subseteq E$ \\
Thresholds limiting the number of TrCPs & $n_{2}^{*} \in \mathbb{N} \cup\{+\infty\}$ \\
Number of TrCP at a given location & $n_{1}^{*} \in \mathbb{N} \cup\{+\infty\}$ \\
\hline
\end{tabular}

Furthermore, according to the RCPs that used to collect information through the inter-urban road network, their deployment are done by ensuring a coverage of the network, minimizing the cost of installation and give the priority to road areas where accidents are mostly caused by the road users. As result, the multiobjective reformulation given as shawn in (12) is then based on three functions $F_{2}, Z_{2}$ and $Z_{3}$ as shown in (6), (7) and (8) as well as constraints $C_{3}, C_{4}$ and $C_{5}$ as shown in (9), (10) and (11). Now, let us define $m_{u, v}$ as a boolean variable that is equal to 1 if there is an edge starting from $u$ to $v$ and 0 otherwise; $\bar{m}_{u, v}$ as a boolean variable that is equal to 1 if there is a way starting from $u$ to $v$ and 0 otherwise; and $a_{u, v}$ that is the number of registered accidents on an edge starting from $u$ to $v$.

$-F_{2}$ : the objective function that represents the deployment cost of TrCPs;

$$
F_{2}=\sum_{u \in P \subseteq \mathbb{N}} \sum_{v \in Q \subseteq E} b_{u, v} y_{u, v}
$$

- $Z_{2}$ allows to prioritize the RCPs where most accidents occur in a given road section;

$$
Z_{2}=\sum_{u \in P \subseteq \mathbb{N}} \sum_{v \in Q \subseteq E} \sum_{k, l \in E} m_{k, v} m_{v, l} a_{u, v} y_{u, v}
$$

- $Z_{3}$ guarantees all vehicles passing through the road network is visible and registered by at least one RCP.

$$
Z_{3}=\sum_{u \in P \subseteq \mathbb{N}} \sum_{v \in Q \subseteq E} \sum_{k, l \in S} \bar{m}_{k, v} \bar{m}_{v, l} y_{u, v}
$$

The constraints $C_{3}, C_{4}$ and $C_{5}$ of the optimization problem are derived as shown in (9), (10) and (11).

$$
C_{3}: \quad \sum_{u \in P} \sum_{v \in Q} \bar{m}_{k, v} \bar{m}_{v, l} y_{u, v} \geq \min \left\{1, \sum_{u \in P} x_{u, k} \sum_{u \in P} x_{u, l} \sum_{v \in Q} \bar{m}_{k, v} \bar{m}_{v, l}\right\}, \forall k, l \in S
$$




$$
\begin{gathered}
C_{4}: \quad \sum_{u \in P} y_{u, v} \leq n_{3}^{*}, \forall v \in Q \\
C_{5}: \quad \sum_{u \in P} \sum_{v \in Q} y_{u, v} \leq n_{4}^{*}, \forall y_{u, v} \in\{0,1\}, \forall u \in P, \forall v \in Q \\
\left\{\begin{array}{l}
\text { Minimize } F_{2} \\
\text { Maximize } Z_{2} \\
\text { Maximize } Z_{3} \\
\text { subject to } C_{3}, C_{4} \text { and } C_{5}
\end{array}\right.
\end{gathered}
$$

Table 2 presents the notations used in the modeling of the optimization problem given as shown in (12).

Table 2. Notations of RCPs

\begin{tabular}{lc}
\hline Variable & Notation \\
\hline Deployment cost for building $R C P_{u}$ at location $v$ & $b_{u, v}>0$ \\
Decision variable to open or not the $R C P_{u}$ at location $v$ & $y_{u, v} \in\{0,1\}$ \\
The number of RCPs candidates & $P$ \\
Set of admissible RCPs positions & $Q \subseteq E$ \\
Thresholds limiting the number of RCPs & $n_{3}^{*}, n_{4}^{*} \in \mathbb{N} \cup\{+\infty\}$ \\
Number of RCP at a given location & $n_{3}^{*} \in \mathbb{N} \cup\{+\infty\}$ \\
Number of registered accidents from $u$ to $v$ & $a_{u, v} \in \mathbb{N}$ \\
\hline
\end{tabular}

Concerning the design Figure 2 illustrates the adopted approach to choose one (or more) method(s) according to the evaluation criteria we chose. For each selected scenario, as basic input parameters, we use:

- An adjacency matrix: Consider $\mathrm{M}$ the adjacency matrix, $M_{i, j}$ designates the possibility of installing a type $i$ control point, at position $j$. It corresponds to the variable $m$ as shown in (5) and (12).

- A cost matrix of installation of Control Point: This is the cost matrix for deploying control point to admissible positions based on their types.

- An accident matrix as input parameters: It defines the number of accidents that occur between the permissible positions of the relay control points (RCP). Thus, $a_{u, v}$ is the number of accidents that occur between point $u$ and point $v$.

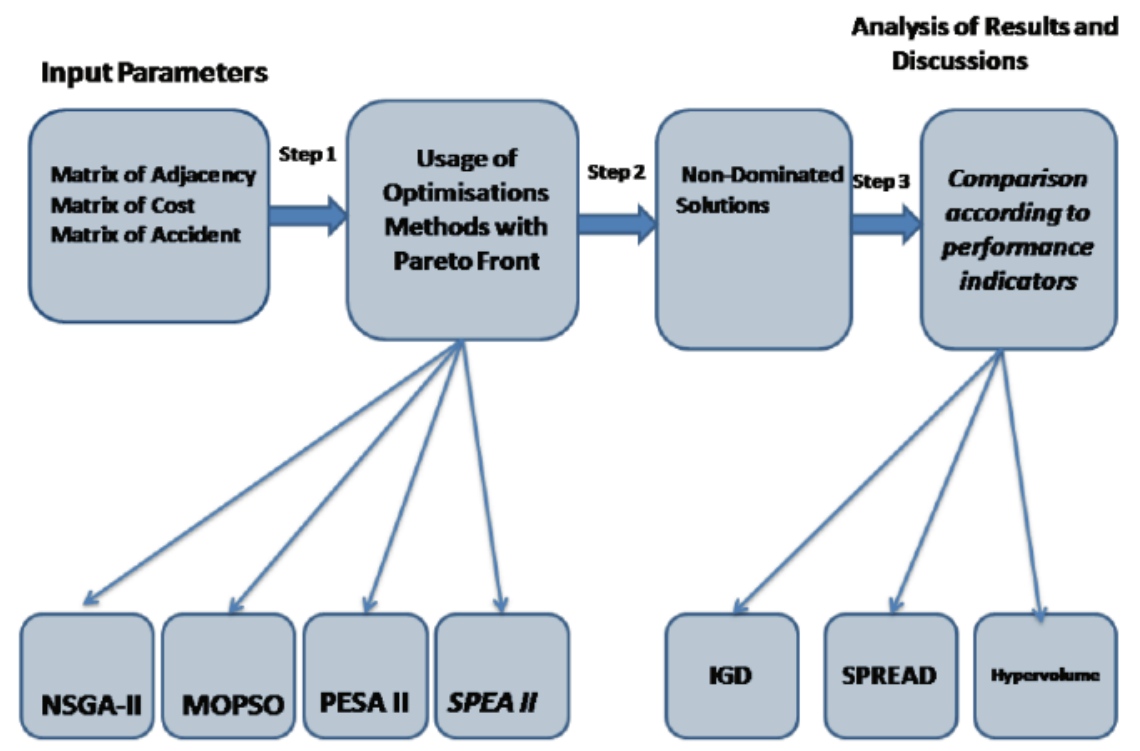

Figure 2. Our proposal design 
Then we use the previously defined methods that will lead to the development of the pareto front. The pareto front allows to highlight for each method, non-dominated solutions. Finally, the chosen performance indicators offer the user the opportunity to select a method in relation to their priorities.

\subsection{Framework}

Here the binary encoding has been used as it can be seen in Figure. 3. Indeed, the objective here is to find the best positions according to the different types of control points. This is in order to minimize the costs of installations by maximizing the coverage of the areas to be monitored and to subserve areas with a high accident rate (case of relay points). Thus, for each chosen scenario, we have an $M \times N$ matrix where $M$ represents the number of control point types and $N$ represents the number of possible positions. The other optimization parameters are given in Table 3.

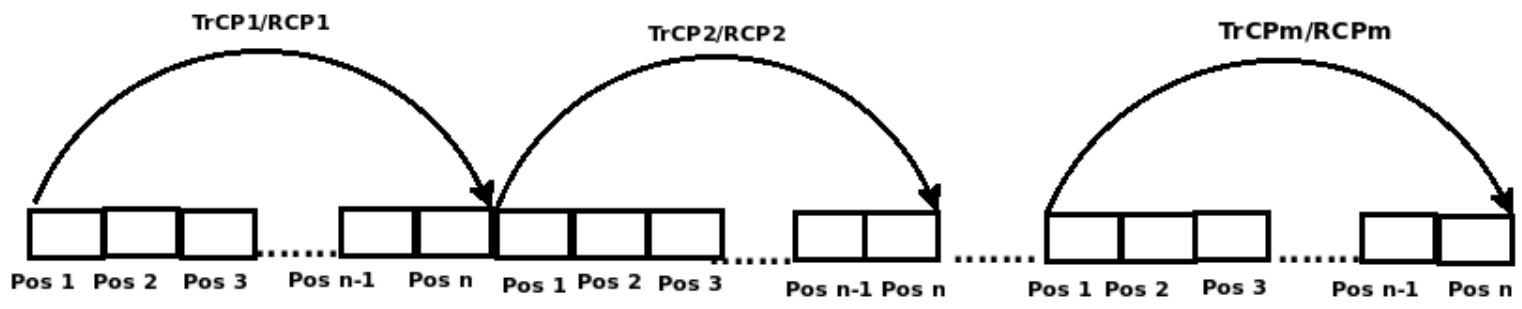

Figure 3. Encoding of TrCPs and RCPs

Table 3. The used parameters of the other optimization methods

\begin{tabular}{cc}
\hline NSGA II, PESA II, SPEA II: Common parameters & Encoding: Binary encoding \\
& Selection:Roulette Wheel Selection \\
Mutation:Gaussian Mutation \\
Crossover:N point crossover, N=2 \\
Population size: 100 \\
Number of iterations:100
\end{tabular}

Probability of crossover $=0.7$, Probability of Mutation $=0.4$

NSGA II:Specifics parameters

PESA II: Specifics parameters

SPEA II: Specifics parameters
Number of Mutants=round (number of population*Probability of Mutation) Mutation Rate $=0.02$

Function: Calculation of crowding distance

Archive size $=100$

Grid Inflation= 0.1

Number of Grids per Dimension $=7$

Archive size $=100$

MOPSO: Parameters

Number of itérations $=100$; Population size $=100$

Repository size $=100$; Number of Grids $=7$

Personal learning coefficient $\mathrm{C} 1=1$; Global learning coefficient $\mathrm{C} 2=2$

Inflation Rate $=0.1$; Leader Selection Pressure $=2$; Deletion Selection Pressure $=2$

\section{PERFORMANCE EVALUATION}

In this section, we present the different results of the deployments of both types of control points. It is for all optimization methods. Then, we present the performance evaluation following the selected criteria. Before we get there, the used experimental values are given in Table 4. The experiments have been conducted in Matlab by considering three scenarios including: five, ten and fifteen admissible positions.

Table 4. Input parameters

Matrix of cost is $\mathrm{c}=$ random([15 75], NbrTypCapt, Nbrpos);

Matrix of adjacency has been set for each scenario;

Matrix of accident $\mathrm{a}=\operatorname{random}([025]$, Nbrpos, Nbrpos $)$ with modifications like a i,j $=$ a j,i

NbrTypCapt is the number of types of control point and Nbrpos represents the number of eligible control points.

In this case, $\mathrm{NbrTypCapt=03}$

Nbrpos $=05,10$ and 15 (admissible positions) 
Given the obtained curves, it would be important to use the tools for evaluating the performance of optimization methods. The evaluation criteria then allow the user to have a logical orientation on the choice to be made. Certainly, there are several performance metrics. The last make it possible to evaluate and compare the quality of the pareto front of the multi objective optimization methods. The work in [41] identified 54 metrics. In addition, according to Okabe et al. [42], these metrics take into account three aspects of the solution set including: the number of solution; the diversify (it is the distribution as well as spread); the convergence (this is the closeness of the theoretical pareto optimal front). Among these performance indicators, we choose the spread, the inverted generational distance and the hypervolume.

- Spread. According to [43, 44], the spread is the metric that gives us a good evaluations results of the approximation. In fact, the spread uses the minimum distance between two successive solutions to the assessment of the distribution of the solution on a given search space. About the comparison of many multi-objective evolutionary algorithms, the lowest value of spread is associated to the best algorithm. Its formula is given as shown in (13).

$$
\left\{\begin{array}{l}
S=\sqrt{\frac{1}{|P|} \sum_{i=1}^{|P|}\left(D_{i}-\bar{D}\right)^{2}} \\
\text { where } \\
\bar{D} \text { is the mean of } D_{i} \\
D_{i}=\min _{j \in P \wedge i \neq j}\left(\sum_{k=1}^{l}\left|f_{k}\left(\overrightarrow{x_{i}}\right)-f_{k}\left(\vec{x}_{j}\right)\right|\right) . \\
\mathrm{P} \text { is the set of the pareto Solution. }
\end{array}\right.
$$

- Inverted generational distance (IGD. It is used to measure the proximity between the evaluated solutions and the optimal pareto front $P^{*}$ [45]. Among many multi-objective evolutionary algorithms, the best algorithm is the one which has the lowest value of IGD. It is defined as given as shown in (14).

$$
\left\{\begin{array}{l}
I G D=\frac{\left(\sum_{i=1}^{|P *|} d_{l}^{*}\left(\overrightarrow{x_{l} *}\right)\right)^{\frac{1}{l}}}{\left|P^{*}\right|} \\
\text { where } \\
d_{l}^{*}\left(\overrightarrow{x_{i}}\right)=\min _{j=1}^{|P|} \sqrt{\sum_{k=1}^{l}\left(f_{k}\left(\overrightarrow{x_{i}^{*}}\right)-f_{k}\left(\overrightarrow{x_{j}^{*}}\right)\right)^{2}} \\
\text { and } \\
\overrightarrow{x_{i}} \text { and } \overrightarrow{x_{j}} \text { are solutions that belong respectively } \\
\text { to } P^{*} \text { and } P
\end{array}\right.
$$

- Hypervolume: It calculate the volume covered by the solutions in a given objective space. It measures both the diversity and convergence as shown in (15). A bigger hypervolume indicator define a better algorithm.

$$
H V=\operatorname{volume}\left(\cup_{i=1}^{|P|} V_{i}\right)
$$

The curves obtained from the optimization methods previously mentioned namely: NSGA-II, PESAII, SPEA-II and MOPSO. Moreover, the previously mentioned scenarios used namely: five eligible positions, ten eligible positions and fifteen eligible positions. Figures $4 \mathrm{a}, 4 \mathrm{~b}$, and $4 \mathrm{c}$ shows the obtained pareto fronts results for TrCP. Furthermore, Figures 5a, 5b, 5c, and 5d shows the pareto fronts results of the RCP with 05 admissible positions. Figures $6 a, 6 b, 6 c$, and $6 \mathrm{~d}$ shows the pareto fronts results of the RCP with 10 admissible positions. Figures 7a, 7b, 7c, and 7d shows the pareto fronts results of the RCP with 15 admissible positions. 


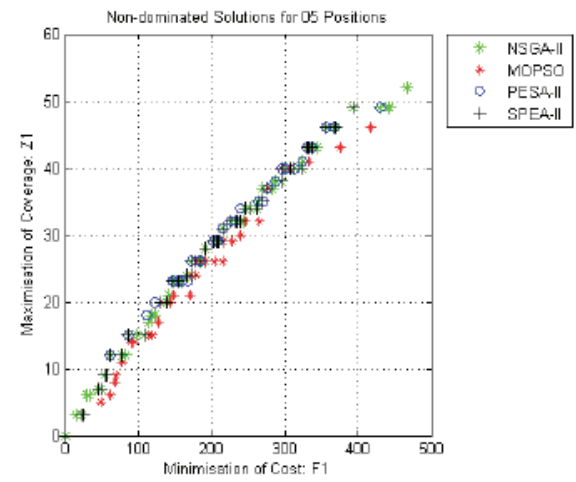

(a)

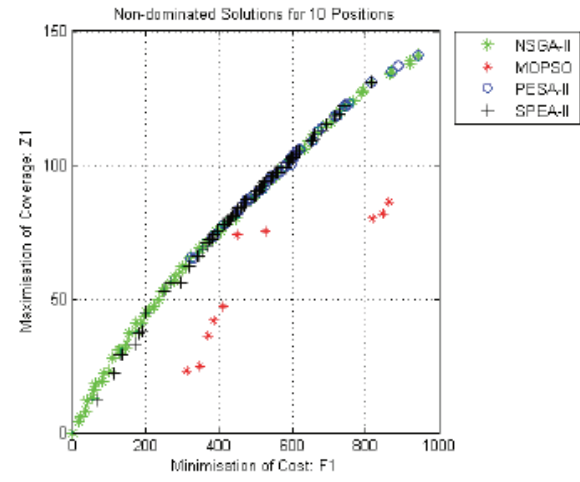

(b)

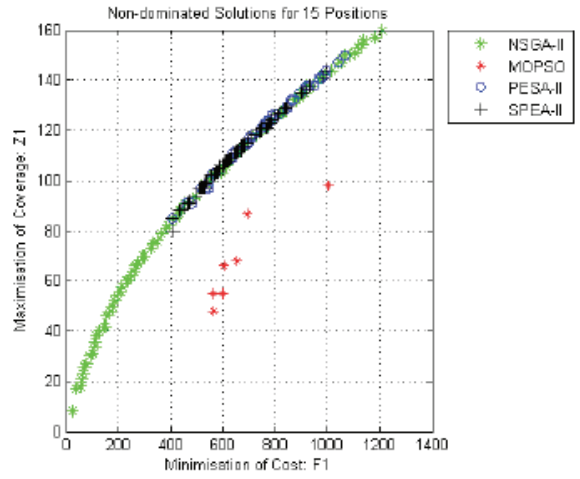

(c)

Figure 4. TrCP pareto's front, (a) 05 positions, (b) 10 positions, (c) TrCP pareto's front

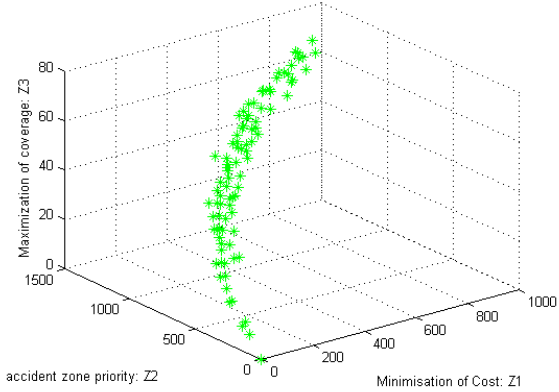

(a)

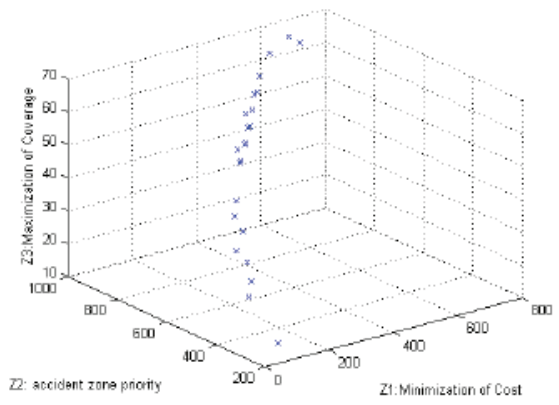

(c)

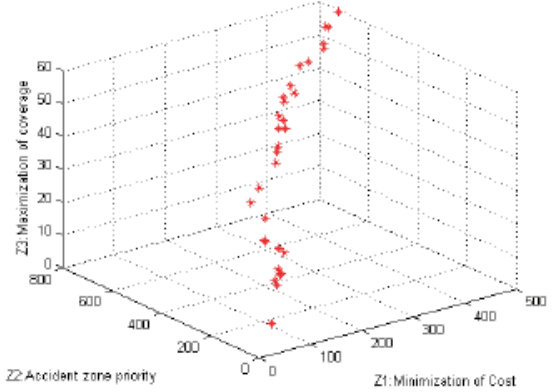

(b)

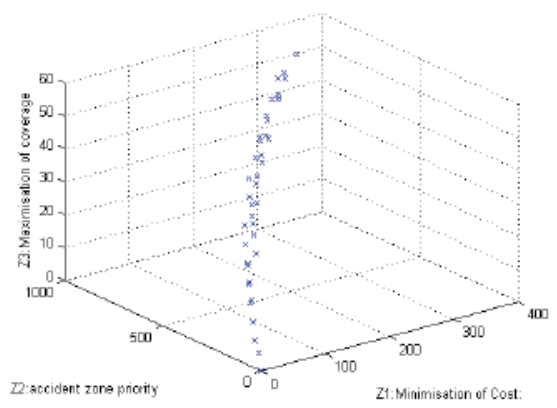

(d)

Figure 5. RCP pareto's front with 05 admissible positions, (a) NSGA-II, (b) NSGA-II, (c) PESA-2, (d) SPEA-2 


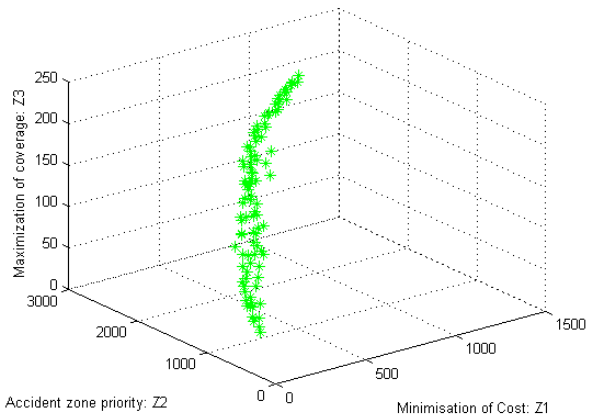

(a)

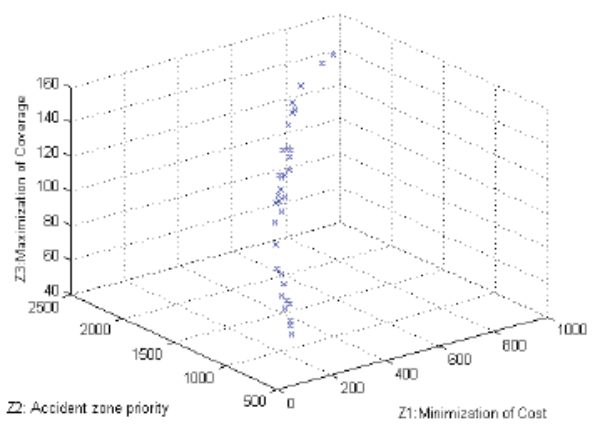

(c)

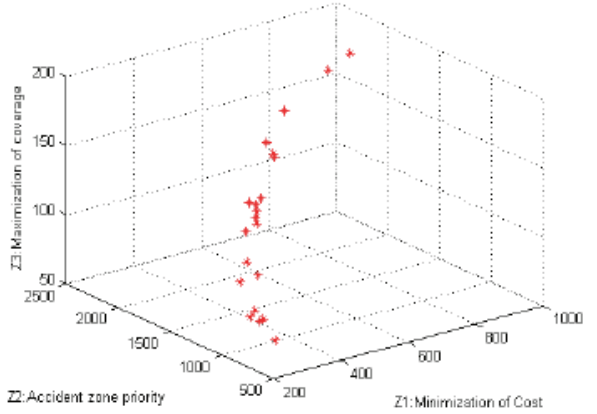

(b)

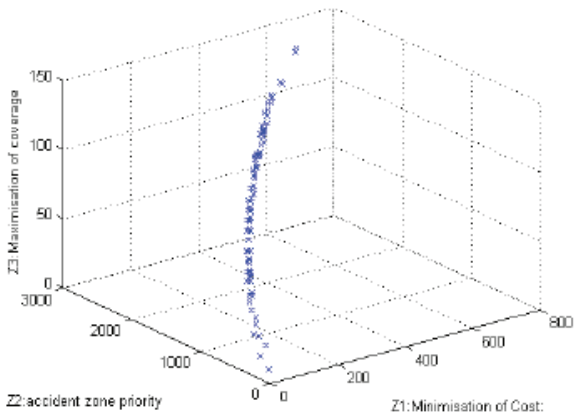

(d)

Figure 6. RCP pareto's front with 10 admissible positions, RCP pareto's front with 10 admissible positions, (a) NSGA-II, (b) MOPSO, (c) PESA-2, (d) SPEA-2

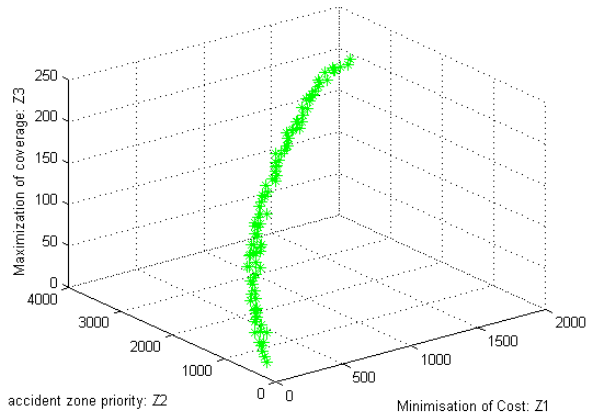

(a)

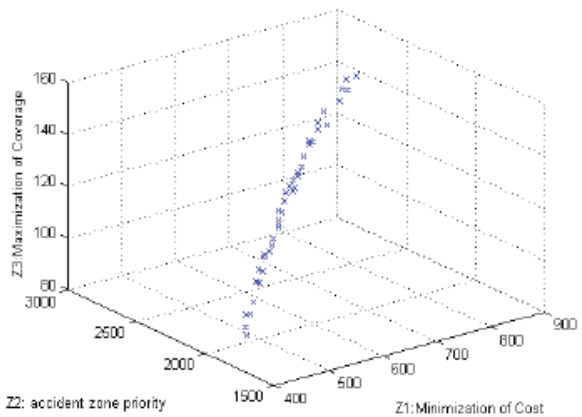

(c)

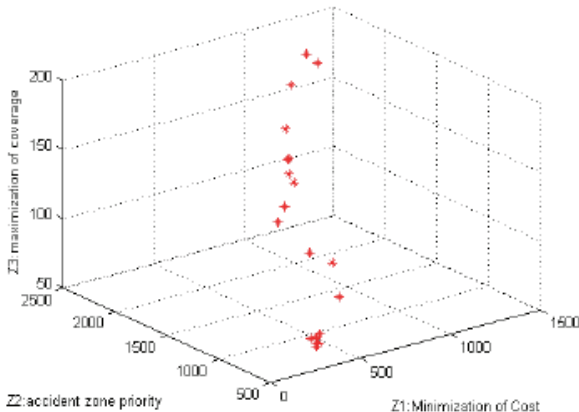

(b)

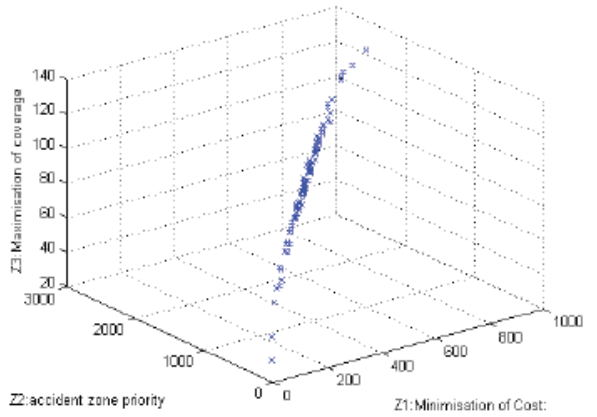

(d)

Figure 7. RCP pareto's front with 15 admissible positions, (a) NSGA II, (b) MOPSO, (c) PESA-2, (d) SPEA-2 
Tables 5, 6, and 7 present the IGD, spread, and hypervolume evaluations respectively of the optimization methods applied to the deployment scenarios of TrCP. Concerning the RCP, the IGD, Spread and Hypervolume are shown in Tables 8, 9, and 10, respectively. With respect to the TrCPs, the comparison of the IGDs shows that for the 05 and 10 admissible points, the PESA-II method is the most appropriate. SPEA-II is best for 15 admissible points. Compared to Spread, the PESA-II method is the best for the deployment for all scenarios. As for the hypervolume, the NSGA-II method is the best. Moreover, with respect to relay checkpoints, the comparison of the IGDs indicates that the SPEA-II is the most appropriate. It is also the best for comparing Spread. As for the hypervolume, the NSGA-II method is the best. Performance indicators have provided more specific orientations to users. Given the large number (i.e., 54) of these indicators, [41], have classified these according to their uses in optimization work. Also, in most of the works in the literature, it appears that Hypervolume is the best criterion of evaluation. This leads us to conclude that the NSGA-II method is the most suitable for the deployment of control points.

Table 5. IGD about TrCPs

\begin{tabular}{lcrrrrrrr}
\hline Numbers of TrCPs & \multicolumn{2}{c}{ NSGA-II } & \multicolumn{2}{c}{ MOPSO } & \multicolumn{2}{c}{ PESA-II } & \multicolumn{2}{c}{ SPEA-II } \\
\hline & Avg & Stdev & Avg & Stdev & Avg & Stdev & Avg & Stdev \\
$\mathbf{0 5}$ & $6.67 \mathrm{e}-01$ & $4.91 \mathrm{e}-01$ & $1.13 \mathrm{e}+00$ & $8.10 \mathrm{e}-01$ & $\mathbf{4 . 9 3 e - 0 1}$ & $\mathbf{4 . 3 1 e - 0 1}$ & $6.11 \mathrm{e}-01$ & $4.46 \mathrm{e}-01$ \\
$\mathbf{1 0}$ & $1.35 \mathrm{e}+00$ & $8.38 \mathrm{e}-01$ & $4.66 \mathrm{e}+00$ & $3.49 \mathrm{e}+00$ & $\mathbf{7 . 3 8 e}-01$ & $\mathbf{6 . 2 7 e - 0 1}$ & $7.54 \mathrm{e}-01$ & $7.79 \mathrm{e}-01$ \\
$\mathbf{1 5}$ & $2.54 \mathrm{e}+00$ & $1.92 \mathrm{e} 00$ & $3.84 \mathrm{e}+00$ & $2.03 \mathrm{e}+00$ & $6.65 \mathrm{e}-01$ & $4.76 \mathrm{e}-01$ & $\mathbf{5 . 2 1 e - 0 1}$ & $\mathbf{5 . 0 5 e - 0 1}$ \\
\hline
\end{tabular}

Table 6. Spread about TrCPs

\begin{tabular}{|c|c|c|c|c|c|c|c|c|}
\hline Numbers of TrCPs & \multicolumn{2}{|c|}{ NSGA-II } & \multicolumn{2}{|c|}{ MOPSO } & \multicolumn{2}{|c|}{ PESA-II } & \multicolumn{2}{|c|}{ SPEA-II } \\
\hline & Avg & Stdev & Avg & Stdev & Avg & Stdev & Avg & Stdev \\
\hline 05 & $1.22 \mathrm{e}+02$ & $1.43 \mathrm{e}+01$ & $8.96 \mathrm{e}+01$ & $1.13 \mathrm{e}+01$ & $5.76 \mathrm{e}-01$ & $5.76 \mathrm{e}+00$ & $2.61 \mathrm{e}+00$ & $1.68 \mathrm{e}+00$ \\
\hline 10 & $2.42 \mathrm{e}+02$ & $4.36 \mathrm{e}+01$ & $2.07 \mathrm{e}+02$ & $8.37 \mathrm{e}+01$ & $1.09 \mathrm{e}+00$ & $1.09 \mathrm{e}+00$ & $2.15 \mathrm{e}+00$ & $1.69 \mathrm{e}+00$ \\
\hline 15 & $3.08 \mathrm{e}+02$ & $7.80 \mathrm{e}+01$ & $1.39 \mathrm{e}+02$ & $8.68 \mathrm{e}+01$ & $1.27 \mathrm{e}+00$ & $1.27 \mathrm{e}+01$ & $2.90 \mathrm{e}+00$ & $1.62 \mathrm{e}+00$ \\
\hline
\end{tabular}

Table 7. Hypervolume about TrCPs

\begin{tabular}{|c|c|c|c|c|c|c|c|c|}
\hline Numbers of TrCPs & \multicolumn{2}{|c|}{ NSGA-II } & \multicolumn{2}{|c|}{ MOPSO } & \multicolumn{2}{|c|}{ PESA-II } & \multicolumn{2}{|c|}{ SPEA-II } \\
\hline & Avg & Stdev & Avg & Stdev & Avg & Stdev & Avg & Stdev \\
\hline 05 & $1.55 e+04$ & $2.66 \mathrm{e}+03$ & $8.32 \mathrm{e}+03$ & $2.54 \mathrm{e}+03$ & $1.18 \mathrm{e}+04$ & $2.35 \mathrm{e}+03$ & $1.15 \mathrm{e}+04$ & $1.75 \mathrm{e}+03$ \\
\hline 10 & $6.93 e+04$ & $1.29 \mathrm{e}+04$ & $2.14 \mathrm{e}+04$ & $1.24 \mathrm{e}+04$ & $3.75 e+04$ & $9.56 \mathrm{e}+03$ & $5.57 e+04$ & $1.17 \mathrm{e}+04$ \\
\hline 15 & $9.92 \mathrm{e}+04$ & $3.52 \mathrm{e}+04$ & $9.23 e+03$ & $7.91 \mathrm{e}+03$ & $2.84 \mathrm{e}+04$ & $8.10 \mathrm{e}+03$ & $3.55 e+04$ & $2.11 \mathrm{e}+04$ \\
\hline
\end{tabular}

Table 8. IGD about RCPs

\begin{tabular}{lcrrrrrrr}
\hline Numbers of RCPs & \multicolumn{2}{c}{ NSGA-II } & \multicolumn{2}{c}{ MOPSO } & \multicolumn{2}{c}{ PESA-II } & \multicolumn{2}{c}{ SPEA-II } \\
\hline & Avg & Stdev & Avg & Stdev & Avg & Stdev & Avg & Stdev \\
$\mathbf{0 5}$ & $1.65 \mathrm{e}+00$ & $1.03 \mathrm{e}+00$ & $1.87 \mathrm{e}+00$ & $1.27 \mathrm{e}+00$ & $2.77 \mathrm{e}+00$ & $1.72 \mathrm{e}+00$ & $\mathbf{9 . 3 6 e - 0 1}$ & $\mathbf{7 . 6 1 e - 0 1}$ \\
$\mathbf{1 0}$ & $2.54 \mathrm{e}+00$ & $1.91 \mathrm{e}+00$ & $5.30 \mathrm{e}+00$ & $4.04 \mathrm{e}+00$ & $3.57 \mathrm{e}+00$ & $3.30 \mathrm{e}+00$ & $\mathbf{1 . 9 9 e}+\mathbf{0 0}$ & $\mathbf{1 . 8 2 e}+00$ \\
$\mathbf{1 5}$ & $2.60 \mathrm{e}+00$ & $1.65 \mathrm{e}+00$ & $4.41 \mathrm{e}+00$ & $3.62 \mathrm{e}+00$ & $2.13 \mathrm{e}+00$ & $1.18 \mathrm{e}+00$ & $\mathbf{1 . 7 7 e}+\mathbf{0 0}$ & $\mathbf{1 . 2 4 e}+\mathbf{0 0}$ \\
\hline
\end{tabular}

Table 9. Spread about RCPs

\begin{tabular}{lcrrrrrrr}
\hline Numbers of RCPs & \multicolumn{2}{c}{ NSGA-II } & \multicolumn{2}{c}{ MOPSO } & \multicolumn{2}{c}{ PESA-II } & \multicolumn{1}{c}{ SPEA-II } \\
\hline & Avg & Stdev & Avg & Stdev & Avg & Stdev & Avg & Stdev \\
$\mathbf{0 5}$ & $4.64 \mathrm{e}+02$ & $4.55 \mathrm{e}+01$ & $3.42 \mathrm{e}+02$ & $2.91 \mathrm{e}+01$ & $1.96 \mathrm{e}+02$ & $3.14 \mathrm{e}+01$ & $\mathbf{3 . 3 4 e + 0 0}$ & $\mathbf{3 . 0 6 e}+\mathbf{0 0}$ \\
$\mathbf{1 0}$ & $8.83 \mathrm{e}+02$ & $1.05 \mathrm{e}+02$ & $6.47 \mathrm{e}+02$ & $7.07 \mathrm{e}+01$ & $3.68 \mathrm{e}+02$ & $8.93 \mathrm{e}+01$ & $\mathbf{2 . 1 6 e + 0 0}$ & $\mathbf{2 . 3 3 e}+\mathbf{0 0}$ \\
$\mathbf{1 5}$ & $1.28 \mathrm{e}+03$ & $9.23 \mathrm{e}+01$ & $1.07 \mathrm{e}+03$ & $1.38 \mathrm{e}+02$ & $3.34 \mathrm{e}+02$ & $5.14 \mathrm{e}+01$ & $\mathbf{9 . 6 1 e - 0 1}$ & $\mathbf{9 . 2 2 e}-\mathbf{0 1}$ \\
\hline
\end{tabular}

Table 10. Hypervolume about RCPs

\begin{tabular}{lcrrrrrrr}
\hline Numbers of RCPs & \multicolumn{2}{c}{ NSGA-II } & \multicolumn{2}{c}{ MOPSO } & \multicolumn{2}{c}{ PESA-II } & \multicolumn{2}{c}{ SPEA-II } \\
\hline & Avg & Stdev & Avg & Stdev & Avg & Stdev & Avg & Stdev \\
$\mathbf{0 5}$ & $\mathbf{3 . 0 2 e + 0 7}$ & $\mathbf{6 . 9 5 e + 0 6}$ & $8.17 \mathrm{e}+06$ & $1.62 \mathrm{e}+06$ & $3.67 \mathrm{e}+06$ & $9.81 \mathrm{e}+05$ & $9.92 \mathrm{e}+06$ & $2.31 \mathrm{e}+06$ \\
$\mathbf{1 0}$ & $\mathbf{1 . 4 8 e + 0 8}$ & $\mathbf{3 . 0 2 e + 0 7}$ & $8.53 \mathrm{e}+07$ & $1.36 \mathrm{e}+07$ & $3.22 \mathrm{e}+07$ & $1.18 \mathrm{e}+07$ & $1.03 \mathrm{e}+08$ & $2.93 \mathrm{e}+07$ \\
$\mathbf{1 5}$ & $\mathbf{4 . 4 9 e + 0 8}$ & $\mathbf{4 . 6 7 e + 0 7}$ & $9.88 \mathrm{e}+07$ & $7.01 \mathrm{e}+06$ & $1.78 \mathrm{e}+07$ & $7.24 \mathrm{e}+06$ & $1.27 \mathrm{e}+08$ & $5.89 \mathrm{e}+07$ \\
\hline
\end{tabular}




\section{CONCLUSION}

Considering the description of our proposed scheme, it appears that ITS can contribute to the regulation of activities in inter-urban road networks in developing countries. The control points are then defined for the collection, RCP and the TrCP of information in this context. We used the multi-objective formulation proposed in previous work for the deployment of control points. We then use the NSGA-II, MOPSO, SPEA-II, PESA-II methods to search among them, the one that would be best indicated for these deployments. Thus, we build the pareto front for these deployments according to three scenarios: 05, 10 and 15 admissible points. We used three performance indicators namely hypervolume, IGD and spread. We noted that the NSGA-II is the preferred method for deploying these control points. Indeed, for all types of control points and for all scenarios, it offers the best results. The future works will consist in proposing an ontology of disturbances that occur in inter-urban road networks.

\section{REFERENCES}

[1] R. Shoukrallah, "Road safety in five leading countries," Journal of the Australasian College of Road Safety, vol. 19, no. 1, 2008, doi: 10.4172/2165-784X.S3-001.

[2] K. Jadaan, E. Al-Braizat, S. Al-Rafayah, H. Gammoh, and Y. Abukahlil, "Traffic safety in developed and developingcountries: Acomparative analysis," Journal of Traffic andLogistics Engineering, vol. 6, no. 1, 2018, doi: 10.18178/jtle.6.1.1-5.

[3] A. A. A. Ari, A. Gueroui, C. Titouna, O. Thiare, and Z. Aliouat, "Resource allocation scheme for 5g c-ran: a swarm intelligence based approach," Computer Networks, pp. 106957, 2019, doi:10.1016/j.comnet.2019.106957.

[4] M. L. Mfenjou, et al., "Methodology and trends for an intelligent transport system in developing countries," Sustainable Computing: Informatics and Systems, vol. 19, pp. 96-111, 2018, doi: 10.1016/j.suscom.2018.08.002.

[5] F. Khedim, N. Labraoui, and A. A. A. Ari, "A cognitive chronometry strategy associated with a re- vised cloud model to deal with the dishonest recommendations attacks in wireless sensor networks," Journal of Network and Computer Applications, vol. 123, pp. 42-56, Sep. 2018, doi: 10.1016/j.jnca.2018.09.001.

[6] M. Babaghayou, et al., "Transmission range changing effects on location privacy-preserving schemes in the internet of vehicles," International Journal of Strategic Information Technology and Applications (IJSITA), vol. 10, no. 4, pp. 33-54, Oct. 2019, doi: 10.4018/IJSITA.2019100103.

[7] J. Zhao, Y. Gao, J. Guo, and L. Chu, "The creation of a representative driving cycle based on intelligent transportation system (its) and a mathematically statistical algorithm: a case study of changchun (china)," Sustainable Cities and Society, vol. 42, pp. 301-313, 2018, doi: https://doi.org/10.1016/j.scs.2018.05.031.

[8] M. Gohar, M. Muzammal, and A. U. Rahman, "SMART TSS: Defining transportation system behavior using big data analytics in smart cities," Sustainable cities and society, vol. 41, pp. 114-119, May 2018, doi: 10.1016/j.scs.2018.05.008.

[9] B. N. Silva, M. Khan, and K. Han, "Towards sustainable smart cities: A review of trends, architectures, components, and open challenges in smart cities," Sustainable Cities and Society, vol. 38, pp. 697-713, Apr. 2018, doi: https://doi.org/10.1016/j.scs.2018.01.053.

[10] C. Peprah, O. Amponsah, and C. Oduro, "A system view of smart mobility and its implications for ghanaian cities," Sustainable Cities and Society, vol. 44, pp. 739-747, Oct.2018, doi: 10.1016/j.scs.2018.10.025.

[11] G. Yadav, S. K. Mangla, S. Luthra, and D. P. Rai, "Developing a sustainable smart city framework for developing economies: An indian context," Sustainable Cities and Society, vol. 47, pp. 101462, May 2019, doi: https://doi.org/10.1016/j.scs.2019.101462.

[12] A. A. A. Ari, A. N. Njoya, D. J. F. Mbogne, W. Abdou, Kolyang, and F. Spies, "Control points deployment in an intelligent transportation system for monitoring inter-urban net- work roadway," Journal of King Saud University - Computer and Information Sciences, Oct 2019, doi: https://doi.org/10.1016/j.jksuci.2019.10.005.

[13] S. Tarapiah, S. Atalla, and R. AbuHania, "Smart on-board transportation management system using gps/gsm/gprs technologies to reduce traffic violation in developing countries," International Journal of Digital Information and Wireless Communications (IJDIWC), vol. 3, no. 4, pp. 96-105, 2013.

[14] F. S. Cabral, et al., "An automatic survey system for paved and unpaved road classification and road 
anomaly detection using smartphone sensor," 2018 IEEE International Conference on Service Operations and Logistics, and Informatics (SOLI), pp. 65-70, Aug. 2018.

[15] P. Patel, Z. Narmawala, and A. Thakkar, "A survey on intelligent transportation system using internet of things," Emerging Research in Computing, Information, Communication and Applications, pp. 231-240, Jan. 2019.

[16] J. Holland, J. Holland, "Adaptation in Natural and Artificial Systems: An Introductory Analysis with Applications to Biology, Control, and Artificial Intelligence," ser. A Bradford book. M.I.T.P., 1992.

[17] A. Kumar, "Encoding schemes in genetic algorithm," International Journal of Advanced Research in IT and Engineering, vol. vol. 2, no. 3, March 2013.

[18] Hancock, A comparison of selection mechanisms, ser. In Handbook of Evolutionary Computation. ds. IOP Publishing and Oxford University Press, Bristol, UK, 1997.

[19] K. Jebari and M. Madiafi, "Selection methods for genetic algorithms," International Journal of Emerging Sciences, vol. 3, no. 4, pp. 333-344, 2013.

[20] P. Kora and S. R. Kalva, "Hybrid bacterial foraging and particle swarm optimization for detecting bundle branch block," SpringerPlus, vol. 4, no. 1, pp. 481, 2015.

[21] P. KoraLi and P. Yadlapalli, "Crossover operators in genetic algorithms: A review," International Journal of Computer Applications, vol. 162, no. 10, Mar. 201, doi: 10.5120/ijca2017913370.

[22] S. Sivanandam and S. Deepa, "Introduction to genetic algorithms," Springer Science Business Media, 2007.

[23] N. Soni and T. Kumar, "Study of various mutation operators in genetic algorithms," International Journal of Computer Science and Information Technologies, vol. 5, no. 3, pp. 4519-4521, 2014.

[24] D. J. Cavicchio, “Adaptive search using simulated evolution,” 1970.

[25] G. B. Fogel and D. B. Fogel, "Continuous evolutionary programming: analysis and experiments," Cybernetics and Syste, vol. 26, no. 1, pp. 79-90, 1995, doi: https://doi.org/10.1080/01969729508927488.

[26] C. A. C. Coello and G. B. Lamont, "Applications of multi-objective evolutionary algorithms," World Scientific, vol. 1, Jan 2004, doi: 10.1142/5712.

[27] K. Deb, A. Pratap, S. Agarwal, and T. Meyarivan, "A fast and elitist multiobjective genetic algo- rithm: Nsga-ii," IEEE transactions on evolutionary computation, vol. 6, no. 2, pp. 182-197, May 2002, doi: 10.1109/4235.996017.

[28] E. Zitzler, M. Laumanns, and L. Thiele, "Spea2: Improving the strength pareto evolutionary algorithm," TIK-report, vol. 103, Jan. 2001.

[29] D. W., N. R. Jerram, J. D. Knowles, and M. J. Oates, "Pesa-ii: Region-based selection in evolutionary multiobjective optimization," [Proceedings of the 3rd Annual Conference on Genetic and Evolutionary Computation. Morgan Kaufmann Publishers Inc.], 2001, pp. 283, Jan. 2001.

[30] J. Kennedy and R. C. Eberhart, "Particle swarm optimization,” [Neural Networks, 1995. Proceed- ings.,] IEEE International Conference on. IEEE, 1995, pp. 1942-1948].

[31] R. C. Eberhart and Y. Shi, "Particle swarm optimization: Developments, applications and ressources," IEEE, 2001.

[32] R. Mendes, J. Kennedy, and J. Neves, “The fully informed particle swarm: simpler, maybe better," IEEE transactions on evolutionary computation, vol. 8, no. 3, pp. 204-210, 2004.

[33] M. Clerc, "The swarm and the queen: towards a deterministic and adaptive particle swarm optimization," Evolutionary Computation, 1999. CEC 99, vol. 3, pp. 1951-1957, 1999, doi: 10.1109/CEC.1999.785513.

[34] R. C. Eberhart and Y. Shi, "Evolving artificial neural networks," Proceedings of the International Conference on Neural Networks and Brain, vol. 1, no. 998, 1998.

[35] R. Mendes, J. Kennedy, and J. Neves, "The fully informed particle swarm: simpler, maybe better," IEEE transactions on evolutionary computation, vol. 8, no. 3, pp. 204-210, Jul. 2004, 10.1109/TEVC.2004.826074.

[36] M. Clerc, "Tribes-un exemple d'optimisation par essaim particulaire sans paramètres de contrôle," Optimisation par Essaim Particulaire (OEP 2003), vol. 64, 2003.

[37] P. J. Angeline, "Evolutionary optimization versus particle swarm optimization: Philosophy and per- formance differences," Int. Conf. on Evolutionary Programming. Springer,pp. 601-610, 1998.

[38] E.-G. Talbi, "A taxonomy of hybrid metaheuristics," Journal of heuristics, vol. 8, no. 5, pp. 541-564, Jan. 2002, doi: 10.1023/A:1016540724870.

[39] C. Zhang, J. Ning, S. Lu, D. Ouyang, and T. Ding, "A novel hybrid differential evolution and particle 
swarm optimization algorithm for unconstrained optimization," Operations Research Letters, vol. 37, no. 2, pp. 117-122, Mar. 2009, doi: https://doi.org/10.1016/j.orl.2008.12.008.

[40] F. Van den Bergh and A. P. Engelbrecht, "A cooperative approach to particle swarm optimization," IEEE transactions on evolutionary computation, vol. 8, no. 3, pp. 225-239, 2004, doi: 10.1109/TEVC.2004.826069.

[41] N. Riquelme, C. Von Lucken, and B. Baran, "Performance metrics in multi-objective optimization," 2015 Latin American Computing Conference (CLEI). IEEE, 2015, pp. 1-11, doi: 10.1109/CLEI.2015.7360024.

[42] T. Okabe, Y. Jin, and B. Sendhoff, "A critical survey of performance indices for multi-objective optimisation," The 2003 Congress on Evolutionary Computation, vol. 2, pp. 878-885, January 2003, doi:10.1109/CEC.2003.1299759.

[43] J. R. Schott, "Fault tolerant design using single and multicriteria genetic algorithm optimization." AIR FORCE INST OF TECH WRIGHT-PATTERSON AFB OH, Tech. Rep., 1995.

[44] A. N. Njoya, et al., "Optimization of sensor deployment using multi-objective evolutionary algorithms," Journal of Reliable Intelligent Environments, vol. 2, no. 4, pp. 209-220, Jun 2016.

[45] W. Abdou, et al., "Using an evolutionary algorithm to optimize the broadcasting methods in mobile ad hoc networks," Journal of Network and Computer Applications, vol. 34, no. 6, pp. 1794-1804, Nov. 2011, doi: https://doi.org/10.1016/j.jnca.2011.01.004.

\section{BIOGRAPHIES OF AUTHORS}

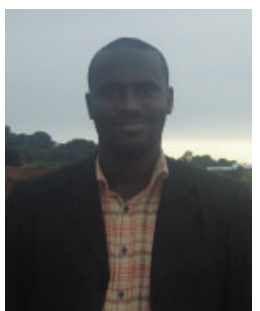

Martin Luther Mfenjou was born in Foumban, Cameroon in 1986. He received his Master of Science (M.Sc.) degree in Computer Engineering from the University of Ngaoundéré in 2010. Currently, He is Assistant Lecturer at the University of Ngaoundéré and he is member of the networks, telecommunications \& services team of the LaRI Lab of the University of Maroua, Cameroon. He is also a Ph.D. Student in Computer Science at the University of Maroua, Cameroon. His current research interests include Intelligent Transportation System in developing countries.

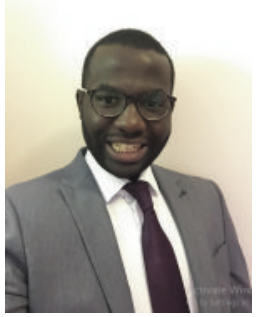

Ado Adamou Abba Ari is a Research Associate at the LI-PaRAD Lab of the Université Paris-Saclay, University of Versailles Saint-Quentin-en-Yvelines, France and Lecturer at the LaRI Lab of the University of Maroua, Cameroon. He received his Ph.D. degree in Computer Science in 2016 from Universite Paris-Saclay in France with the higher honors. He also received the Master Degree of Business Administration (MBA)in 2013, the Master of Science (M.Sc.) degree in Computer Engineering in 2012 and the Bachelor of Science (B.Sc.) degree in Mathematics and Computer Science in 2010 from the University of Ngaoundéré, Cameroon. He served/serving on several journals and conferences program and reviewing committees. Thus, he achieved the outstanding reviewer status with the Elsevier Computer Networks. Moreover he his recognized reviewer of a number of journals including IEEE Transactions on Intelligent Transportation Systems, IEEE Access, Information Sciences, Journal of Network and Computer Applications, Computer Communications, Remote Sensing, Sensors, Electronics, Telecommunication Systems, Wireless Personal Communications, Sustainable Computing: Informatics and Systems, Journal of Ambient Intelligence and Humanized Computing, etc. His current research is focused on bio-inspired computing, Wireless Networks, IoT, 5G and the Cloud Radio Access Network.

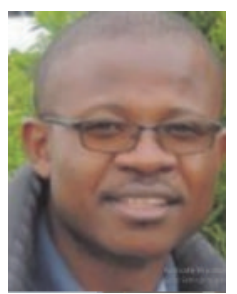

Arouna Ndam Njoya received the Master of Engineering (M.Eng.) degree in telecommunications from the National Advanced School of Engineering, University of Yaoundé I, Cameroon in 2009, the Master of Science (M.Sc.) degree in computer engineering and the Ph.D. degree in computer science both from the Faculty of Sciences of the University of Ngaoundéré, Cameroon, respectively in 2012 and 2018. He is actually serving as Lecturer at the Department of Computer Engineering, University Institute of Technology, University of Ngaoundéré. Dr. Arouna Ndam Njoya served/serving as a reviewer for various journals international conferences. His research work is mainly on the fields of combinatorial optimization, evolutionary computation, Big data, IoT and coverage maintaining in WSNs. 


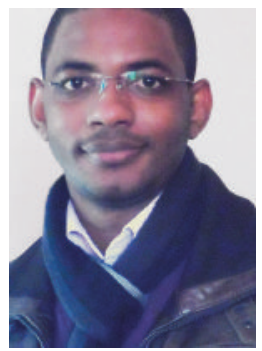

Wahabou Abdou is an associate professor in computer science at the at the University of Burgundy, Dijon France. He received his Ph.D. degree in computer science, the Master of Science (M.Sc.) in computer's services and mobile networks from the University of Franche-Comté, France, respectively in 2011 and 2008. Prior to this, he also received the Master of Engineering (M.Eng.) degree in telecommunication from the Polytechnic Institute of Conakry, Guinea in 2007, the Bachelor of Engineering (B.Eng.) in networks from the African Institute of Informatics, Cameroon in 2005 and the two years technological university diploma in computer engineering from the University of Ngaoundéré, Cameroon in 2003. Attached to the CombNet (Combinatorics and Networks) team of LE2I (Laboratory of Electronics, Computer and Image), his research interests include issues related to communications (routing, broadcast, quality of service) in ad hoc networks (MANETs, VANETs), autonomous computing, distributed algorithmics, algorithmic evolutionary (including genetic algorithmic) and multi-objective optimization.

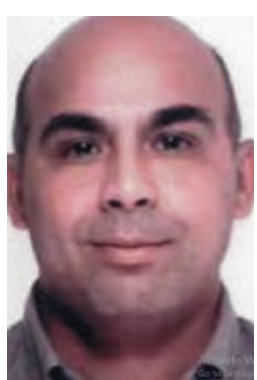

Abdelhak (Mourad) Gueroui is an associate professor in computer engineering at the University of Versailles Saint-Quentin-en-Yvelines, France. He received his M.Sc. degree from Université Paris 6 and his Ph.D. in networking and computer engineering in 2000 from the University of Versailles Saint-Quentin-en-Yvelines, France. He has authored more than 100 publications in international conferences and journals, as well as book chapters, including ACM, IEEE, ELSEVIER and has both chaired and served in numerous program committees in prestigious international conferences. $\mathrm{He}$ received several awards, including best papers and serves/served on several journals and conferences executive committees. His research interests are in the area of wireless networks (WATM, WIMAX, LTE, 5G, Cloud Computing, WLAN, MESH, VANET and WSNs), particularly performance evaluation and QoS provisioning. 